\title{
An instance data repository for the round-robin sports timetabling problem*
}

\author{
David Van Bulck ${ }^{\dagger} \quad$ Dries Goossens $\ddagger \quad$ Jörn Schönberger $§ \quad$ Mario Guajardo $q$
}

\begin{abstract}
The sports timetabling problem is a combinatorial optimization problem that consists of creating a timetable that defines against whom, when, and where teams play games. This is a complex matter since real-life sports timetabling applications are typically highly constrained. The vast amount and variety of constraints and the lack of generally accepted benchmark problem instances make that timetable generators are often tested on just one or two specific instances. This is problematic since few algorithmic insights are gained. To mitigate this issue, this paper provides a problem instance repository containing over 40 different instance types, covering artificial and real-life problem instances. The construction of such a repository is not trivial since there are dozens of constraints that need to be expressed in a standardized format. For this, our repository relies on RobinX, an XML-supported classification framework. The resulting repository provides a (non-exhaustive) overview of most real-life sports timetabling applications published over the last five decades. For every problem, a short description highlights the most distinguishing characteristics of the problem. The repository is publicly available and will be continuously updated as new instances or better solutions become available.
\end{abstract}

Index terms - Timetabling, Sports scheduling, Benchmark, XML, RobinX

\section{Introduction}

Researchers have been creating timetables, also called schedules, for sports tournaments since the 1970's (e.g. Ball and Webster (1977)). Particularly popular are round-robin timetables in which every team plays against every other team a fixed number of times. Since the seventies, there has been a strong increase in the amount of sports timetabling contributions. This increase has been accompanied by many newly proposed constraints and objective functions, often originating from real-life applications. Table 1 illustrates the increase in contributions over the last five decades. Since no set of benchmark problem instances is publicly available, it is hard to assess the algorithmic performance of the various solution methods proposed. Indeed, as contributions in the literature are often tested on just one or two specific problem instances, little algorithmic understanding is gained. One notable exception is the traveling tournament problem (Easton et al., 2001), which minimizes the total team travel in the timetable. For this problem, substantial algorithmic progress has been reported after Trick (2010) made a set of problem instances publicly available.

Nurmi et al. (2010) are the first to set up a (plain text-only) file format to store more complex instances. They also propose a set of artificial and real-world instances together with the best solutions found so far. This format, however, has limited utility with respect to the ease of data manipulation and is not extensible towards several real-world problems. Recently, Van Bulck et al. (2020) proposed a more advanced XML-supported three-field notation, coined RobinX, to describe and exchange sports timetabling instances and solutions. RobinX can store nearly every constraint found in the literature and is complemented with a C++-library to read, write, and evaluate these XML files.

The contribution of the current paper is to use RobinX to describe a wide variety of problem instances and solutions from the literature. In addition, since these instances include most real-life applications published over the last five decades, the paper serves as a guide to the literature on the practice of sports timetabling. All the resulting XML files are publicly available on www. sportscheduling. ugent. be/RobinX. Similar initiatives have revolutionized algorithmic development in other research disciplines such as school timetabling (Post et al., 2012, 2014) and nurse rostering (Kingston et al., 2018). We hope that this paper encourages researchers to benchmark their algorithms on a broader set of instances and to share their own data sets with the research community.

\footnotetext{
${ }^{*}$ This is the peer-reviewed author-version of https://journals.sagepub.com/doi/full/10.1177/0258042X20912108, published in Management and Labour Studies.

${ }^{\dagger}$ Ghent University, Faculty of Economics and Business Information, E-mail: david.vanbulck@ugent.be

${ }^{\ddagger}$ Ghent University, Faculty of Economics and Business Information

$\S$ Technische Universität Dresden, Institute of Transport and Economics

『NNH Norwegian School of Economics, Department of Business and Management Science
} 
Table 1: Number of (round-robin) sports timetabling contributions related to real-life applications. Numbers are mainly based on title and abstract selection from Knust (2018).

\begin{tabular}{ccccc}
\hline $1970-1979$ & $1980-1989$ & $1990-1999$ & $2000-2009$ & $2010-2018$ \\
\hline 3 & 3 & 12 & 20 & 23
\end{tabular}

Table 2: A compact double round-robin timetable for a single league with 6 teams. Each game is represented by an ordered pair in which the first element is the home team, and the second element is the away team.

\begin{tabular}{cccccccccc}
\hline$s_{1}$ & $s_{2}$ & $s_{3}$ & $s_{4}$ & $s_{5}$ & $s_{6}$ & $s_{7}$ & $s_{8}$ & $s_{9}$ & $s_{10}$ \\
\hline$(1,2)$ & $(2,5)$ & $(2,4)$ & $(2,3)$ & $(6,2)$ & $(2,1)$ & $(5,2)$ & $(4,2)$ & $(3,2)$ & $(2,6)$ \\
$(3,4)$ & $(4,1)$ & $(1,6)$ & $(5,1)$ & $(4,5)$ & $(4,3)$ & $(1,4)$ & $(6,1)$ & $(1,5)$ & $(5,4)$ \\
$(5,6)$ & $(6,3)$ & $(5,3)$ & $(6,4)$ & $(1,3)$ & $(6,5)$ & $(3,6)$ & $(3,5)$ & $(4,6)$ & $(3,1)$
\end{tabular}

The remainder of this paper is as follows. First, Section 2 formally defines the sports timetabling problem, and introduces the most common sports timetabling terminology. Thereafter, Section 3 summarizes the main characteristics of RobinX. Section 4 presents our problem repository and describes the most prominent properties of each data set. Finally, Section 5 presents some conclusions.

\section{Terminology}

The input of a sports timetabling problem consists of a set of teams $T$ with $|T|=n$, a set of time slots $S$, and a multiset of games $G$. Time slots represent periods in time such as half days, days, or weekends in the season. A team can play at most one game per time slot in $S$; a team has a bye if it does not play at all in that time slot. The multiset of games $G$ consists of ordered pairs $(i, j)$ in which $i \in T$ is the home team providing the venue where the game is played, and $j \in T$ is the away team. For convenience, we denote with $g_{i, j}$ the multiplicity of the ordered pair $(i, j) \in G$, i.e. $g_{i, j}$ gives the number of home games $i$ has to play against $j$. Similarly, we denote with $g_{i}$ the total number of games in $G$ involving team $i$.

In a $k$ round-robin tournament (kRR), $g_{i, j}+g_{j, i}$ equals $k$ for each $i, j \in T$ with $i \neq j$. Similarly, in a $k$ bipartite round-robin tournament (kBRR), the teams in $T$ can be partitioned into two groups $T_{1} \cup T_{2}$ in such a way that $g_{i, j}+g_{j, i}$ equals $k$ if $i \in T_{1}$ and $j \in T_{2}$, and 0 otherwise. In multi-league $k$ round-robin tournaments, the teams in $T$ can be partitioned into different leagues in such a way that $g_{i, j}+g_{j, i}$ equals $\mathrm{k}$ if $i \in T$ and $j \in T \backslash i$ are in the same league, and 0 otherwise. In any other case, we refer to the tournament structure as a non round-robin tournament (NRR). A tournament is compact if the number of available time slots $|S|$ is no more than the minimum number required to play all games in that tournament. Otherwise, the tournament is time-relaxed. As an example, in a kRR the minimum number of time slots to play all games equals $k(n-1)$ if $n$ is even, and $k n$ if $n$ is odd. A timetable maps each game in $G$ to a time slot $s \in S$ such that no team plays more than one game per time slot. Table 2 gives an example of a timetable for a compact $2 \mathrm{RR}$. In a $\mathrm{kRR}$ with $k>1$, the season is often split into $k$ intervals, i.e. a series of consecutive time slots of length $|S| / k$, that each contain a 1 RR and in which the home-away status of mutual games for each pair of teams alternates between consecutive intervals. We call a timetable that follows this format phased and consider the following additional symmetry structures (see Table 3). In the mirrored timetable format, the opponents in each interval are identical to the opponents of the previous interval (see e.g. Table 2. In the inverse system, intervals are played in the reversed order of the previous interval. In the English system, the opponents in the first time slot of an interval are the same as in the last time slot of the previous interval. For all other time slots, the opponents of the $l$-th time slot of the interval correspond with opponents of the $(l-1)$-th time slot of the previous interval. Finally, in the French system, the opponents in the last time slot of an interval correspond with the opponents in the first time slot of the previous interval. For all other time slots, the opponents of the $l$-th time slot in in an interval correspond with the opponents in the $(l+1)$-th time slot of the previous interval. An overview of symmetry structures in European top football (also called soccer) tournaments can be found in (Goossens and Spieksma, 2012).

A team has a home stand if it plays multiple home games in a row and is on a road trip when it plays multiple away games in a row. If a team plays a game with the same home-away status as its previous game, independently of the total number of time slots between the two games, we say it has a break. As an example, team 2 in Table 2 has a home break in time slot $s_{3}$ and $s_{4}$ and has a home stand starting in $s_{2}$ and ending in $s_{4}$. When a team first plays against team $i$, and immediately thereafter against team $j$, we say that team $i$ gives a carryover effect (COE) to team $j$. If we denote with $c_{i, j}$ the number of carryover effects that team $i$ gives to team $j$, then the carryover 
Table 3: An illustration of different symmetry schemes (based on Goossens and Spieksma (2012)). The number of time slots in each of the $k$ intervals is $n=|S| / k$. Each number in the table corresponds with the opponents of a time slot.

\begin{tabular}{lllllllllllll}
\hline & \multicolumn{1}{c}{ Interval $i$} & \multicolumn{1}{c}{ Interval $i+1$} \\
\hline Mirrored & 1 & 2 & 3 & $\ldots$ & $n-1$ & $n$ & 1 & 2 & 3 & $\ldots$ & $n-1$ & $n$ \\
Inverse & 1 & 2 & 3 & $\ldots$ & $n-1$ & $n$ & $n$ & $n-1$ & $n-2$ & $\ldots$ & 2 & 1 \\
English & 1 & 2 & 3 & $\ldots$ & $n-1$ & $n$ & $n$ & 1 & 2 & $\ldots$ & $n-2$ & $n-1$ \\
French & 1 & 2 & 3 & $\ldots$ & $n-1$ & $n$ & 2 & 3 & 4 & $\ldots$ & $n$ & 1
\end{tabular}

effects value (COE-value) of a timetable is defined as $\sum_{i \in T} \sum_{j \in T \backslash i} c_{i, j}^{2}$ (Russell, 1980). Note that the COE-value is a cyclical concept: it also considers the carryover from a team's last game to its first game. For fairness reasons, it is sometimes requested that the COE-value of a team group is as low as possible. For example, if $i$ is very strong team, it could be argued that $j$ has an advantage since $j$ 's opponent is more likely to be weakened or injured. For a complete overview of sports timetabling terminology, we refer to (Kendall et al., 2010).

\section{A three-field notation for sports timetabling}

This section briefly outlines the three-field notation $(\alpha / \beta / \gamma)$, as proposed in (Van Bulck et al., 2020), to describe different variants of the sports-timetabling problem.

First, the $\alpha$-field uses three parameters to describe the different tournament formats. The first parameter distinguishes multi-league problems, $k$-round robin tournaments, $k$-bipartite round-robin tournaments, and nonround-robin tournaments. The second parameter differentiates between compact and time-relaxed timetables. By default, the third parameter assumes that the tournament does not require any symmetry at all. However, in case of a kRR with $k>1$, the third parameter considers the phased, mirrored, inverse, English, and French symmetry structure.

Second, the $\beta$-field lists around 30 constraint types partitioned into five constraint groups that classify the vast majority of the constraints from the literature. Capacity constraints enforce a team to play home or away and regulate the total number of games played by a given set of teams. Game constraints enforce or forbid specific game to time slot assignments. Next, break constraints regulate the frequency and timing of breaks in a tournament. Fairness constraints distribute timetable inconveniences over all teams or increase the attractiveness of the league. Finally, separation constraints regulate the number of time slots between consecutive games involving the same teams and regulate the symmetry of the timetable. Constraints are either hard or soft and have a nonnegative penalty weight: the total cost induced by a constraint is (a transformation of) the amount of violation multiplied with the penalty weight. The cost of violated hard constraints contributes to the infeasibility value of a timetable whereas the cost of violated soft constraints is included into the objective value of a timetable. A timetable is feasible if and only if it has an infeasibility value of zero.

Lastly, the $\gamma$-field refers to the objective function in use. If no objective function is provided, the problem reduces to a constraint satisfaction problem in which the sole purpose is to find a timetable respecting all hard constraints. Besides, this field considers the optimization of breaks, travel distance, costs or revenues, COE-value, and soft constraint violations. The latter minimizes the cost resulting from violated soft constraints while still respecting all hard constraints. For all other objectives, all constraints must be hard.

\section{Benchmark instances}

This sections uses the three-field notation outlined in Section 3 to describe a wide variety of sports timetabling instances that had previously appeared in the literature. We group single-league problem instances along their objective function, and dedicate a special section for multi-league instances. A more sophisticated query tool and all XML files are available on our website (www.sportscheduling.ugent.be/RobinX). In the remainder of this paper, we refer to the total number of requirements imposed by the league organizers as the total number of constraints. However, each requirement could have multiple constraints. Table 4 summarizes all problems described in this section. 
Table 4: Overview of the different problem instances covered by the repository. For the multi-league instances, the third column denotes the range of the number of teams per league.

\begin{tabular}{|c|c|c|c|}
\hline Paper reference & No. instances & No. teams & Sport (country) \\
\hline \multicolumn{4}{|l|}{ Constraint satisfaction } \\
\hline Nemhauser and Trick (1998) & 1 & 9 & Basketball (USA) \\
\hline Zhang (2002) & 1 & 11 & Basketball (USA) \\
\hline Briskorn and Drexl (2009a) & 69 & $4-40$ & Artificial \\
\hline Nurmi et al. (2010) & 2 & $14-16$ & Artificial \\
\hline \multicolumn{4}{|l|}{ Break optimization } \\
\hline Della Croce and Oliveri (2006) & 1 & 18 & Football (Italy) \\
\hline Nurmi et al. (2010) & 18 & $8-16$ & Artificial \\
\hline Hausken et al. (2012) & 1 & 16 & Football (Norway) \\
\hline Nurmi et al. (2013) & 1 & 16 & Ice hockey (Finland) \\
\hline Durán et al. (2017) & 1 & 9 & Football (South America) \\
\hline \multicolumn{4}{|l|}{ Travel optimization } \\
\hline Easton et al. (2001) & 7 & $4-16$ & Artificial \\
\hline Easton et al. (2001) & 19 & $4-40$ & Artificial \\
\hline Urrutia and Ribeiro (2006) & 19 & $4-40$ & Artificial \\
\hline Ribeiro and Urrutia (2007) & 1 & 24 & Artificial \\
\hline Melo et al. (2009) & 20 & $18-20$ & Artificial \\
\hline Uthus et al. (2009) & 19 & $4-40$ & Artificial \\
\hline Trick (2010) & 9 & $16-32$ & Artificial \\
\hline Trick (2010) & 4 & $6-30$ & Artificial \\
\hline Hoshino and Kawarabayashi (2011b) & 2 & $12-30$ & Artificial \\
\hline Bonomo et al. (2012) & 1 & 12 & Volleyball (Argentina) \\
\hline Hoshino and Kawarabayashi (2012) & 38 & $4-40$ & Artificial \\
\hline Uthus et al. (2012) & 19 & $4-40$ & Artificial \\
\hline \multicolumn{4}{|l|}{ Cost and revenue optimization } \\
\hline Durán et al. (2007) & 2 & 20 & Football (Chile) \\
\hline Uthus et al. (2008) & 10 & $8-16$ & Artificial \\
\hline Durán et al. (2012) & 1 & 11 & Football (Chile) \\
\hline \multicolumn{4}{|l|}{ Carryover effects } \\
\hline Kidd (2010) & 14 & $4-30$ & Artificial \\
\hline Guedes and Ribeiro (2011) & 69 & $4-24$ & Artificial \\
\hline Günneç and Demir (2018) & 6 & $8-18$ & Football (Turkey) \\
\hline \multicolumn{4}{|l|}{ Soft constraint } \\
\hline Schönberger et al. (2004) & 57 & 10 & Table tennis (Germany) \\
\hline Wright (2006) & 1 & 10 & Basketball (New Zealand) \\
\hline Bartsch et al. (2006) & 3 & $10-18$ & Football, handball (Germany, Austria) \\
\hline Rasmussen (2008) & 1 & 12 & Football (Denmark) \\
\hline Goossens and Spieksma (2009) & 3 & 18 & Football (Belgium) \\
\hline Kyngäs and Nurmi (2009a) & 1 & 12 & Ice hockey (Finland) \\
\hline Kyngäs and Nurmi (2009b) & 1 & 14 & Ice hockey (Finland) \\
\hline Lewis and Thompson (2011) & 10 & $12-18$ & Rugby (Wales) \\
\hline Recalde et al. (2013) & 1 & 12 & Football (Ecuador) \\
\hline Larson and Johansson (2014) & 10 & 14 & Handball (Sweden) \\
\hline Nurmi et al. (2015) & 1 & 14 & Ice hockey (Finland) \\
\hline Cocchi et al. (2018) & 1 & 14 & Volleyball (Italy) \\
\hline Van Bulck et al. (2019) & 44 & $13-15$ & Indoor football (Belgium) \\
\hline \multicolumn{4}{|l|}{ Multi-league } \\
\hline Kendall (2008) & 4 & $20-24$ & Football (UK) \\
\hline Schönberger (2017) & 396 & 10 & Artificial \\
\hline
\end{tabular}




\subsection{Constraint satisfaction problems}

In this group of instances, all constraints are hard and no objective function is given. In other words, it is required to construct a timetable with an infeasibility value of zero.

A first instance originates from (Nemhauser and Trick, 1998) and consists of constructing a compact 2RR with 9 teams for the 1997-1998 season of the Atlantic Coast basketball conference (ACC). All games need to be scheduled over a nine-week period in which each week has one weekday and one weekend time slot. In total, there are nine different hard constraints, of which the most prominent are the following. First off, to separate games between the same pair of teams, the timetable must follow a symmetry closely related to the mirrored scheme. Since teams value weekend slots higher than weekday slots, each team must have four home games, four away games, and one bye during weekend time slots. In order to promote the team and attract new players, teams can have at most three away games in the first five weekends, and no team can play consecutively away on the last two time slots. Moreover, teams can play at most two home or away games in a row, there are limits on the number of consecutive games against strong opponents, and there are some fixed games. A closely related instance consists of constructing a timetable for the 2002 regular season of the BigTen basketball tournament (Zhang, 2002). However, this instance contains 11 teams that compete in a partial 2RR in which each team plays at least once and at most twice against every other team. More in particular, each team plays exactly 16 games during a nine-week period in which each week has one weekday and one weekend time slot. There are only two hard constraints. First, as in the ACC, it is required that each team plays the same number of home and away games during weekday and weekend time slots. Second, a team can play at most two home or away games in a row.

Another set of instances concerns the question whether a compact group-changing or group-balanced timetable exists. Given $|T|$ teams and a partitioning of these teams into $g$ equally-sized strength groups, Briskorn (2009) calls a timetable 'group-changing' if no team plays against teams of the same strength group in two consecutive games. Similarly, a timetable is ' $g$-group-balanced' if no team plays more than once against teams of the same strength group within $g$ consecutive games. Our database contains several instances and known solutions for $|T| \leq 40$ and $g \leq 20$.

Finally, we included two artificially generated instances proposed in (Nurmi et al., 2010). The first instance contains 14 teams and the task is to construct a compact 2RR under two hard constraints. First, there must be at least 7 time slots before two teams meet again. Second, in each time slot, there are four teams that cannot play home and four teams that cannot play away. The second instance is based on the Finnish major ice hockey league for players under 20 years of age (see (Nurmi et al., 2013)). In this instance, 16 teams compete in a 3RR for which the home teams in the third meeting are fixed beforehand. Since there are 57 time slots available but only 45 time slots are strictly needed, the tournament is time-relaxed. There are two hard constraints: some teams must play home or away in a given round and all teams are paired with another team with whom they cannot play simultaneously at home.

\subsection{Break optimization}

In the constrained minimum break problem, we try to minimize the total number of breaks under a series of hard constraints.

A first instance is derived from the 2003-2004 season of the Italian major football league (Della Croce and Oliveri, 2006) in which 18 teams play a compact 2RR. The objective is to minimize the total number of breaks, while considering the following hard constraints. First of, the best four teams of the previous year cannot play against each other in the first and last three time slots. Somewhat similarly, some teams share a stadium and should therefore not play simultaneously at home, nor should they play against each other in the first or last three time slots. Moreover, a team cannot play more than two consecutive home or away games. Since Italian law forbids the presence of a monopoly, the Italian major football league collaborates with two television companies that each own the exclusive rights to broadcast all home games of a subset of teams. In order to reduce the costs of renting additional channels, the broadcasters demand that in each time slot half of its assigned teams play a home game. Della Croce and Oliveri (2006) also mention that teams have preferences to play home or away in particular time slots, however our repository ignores this constraint since no data is available.

The Norwegian 2009 and 2010 major football league (Hausken et al., 2012) instances consist of 16 teams that compete in a compact phased 2RR. The objective is to minimize the total number of breaks, while considering the following hard constraints. First, for fairness reasons, there cannot be a break in the second and final time slot. Second, television broadcasters require that there is at least one game between two attractive teams in every time slot. Similarly, it is required that there are at least four time slots between two games with the same pair of opponents. Some pairs of teams cannot play at home simultaneously since they share venues. Finally, some teams cannot play at home or away in given time slots. We note that Hausken et al. (2012) also consider some problem 
extensions in which a number of soft constraints are additionally considered. However, since not all data for these soft constraints is available, our repository does not include these problem variants.

The instance of the South American qualification tournament for the 2018 FIFA World Cup (Durán et al., 2017) consists of nine teams that compete in a compact $2 \mathrm{RR}$ with 18 time slots that are partitioned into nine 'time pairings'. Although there are only a few days between time slots of the same time pairing, there are several months between time slots of different time pairings. Given this long period, the objective is to minimize breaks that occur when a team plays two home or two away games within a time pairing. Moreover, the following hard constraints must be respected. For fairness reasons, the total number of generalized breaks per team is limited and no bottom team can play consecutively against top teams. Besides, Durán et al. (2017) experiment with many different symmetric schemes of which the final proposed timetable follows the French scheme.

Nurmi et al. (2010) propose a set of 18 artificial instances in which the number of teams ranges from 8 to 16. In 17 out of the 18 instances, the tournament in question is a compact $2 \mathrm{RR}$, whereas in the other instance the tournament is a compact $1 \mathrm{RR}$. In all instances, the objective is to minimize the total number of breaks while considering one or more of the following hard constraints. A first constraint enforces to have at least a given minimal number of time slots to last between two consecutive games of a team. Additionally, the constraints may include capacity constraints stating that a team cannot play at home or away in a given time slot, or that two teams cannot play home simultaneously. Finally, Nurmi et al. (2010) also propose an artificial instance based on the Finnish major ice hockey league for players under 20 years of age (see (Nurmi et al., 2013)). This instance is similar to the one explained in Section 4.1, but there is only one pair of teams that cannot play home simultaneously and the objective is to minimize the number of breaks. Moreover, there are additionally fixed games and at least 12 time slots must pass before two teams can meet again.

\subsection{Travel optimization}

The traveling tournament problem (TTP, Easton et al. (2001)) is an artificial yet popular sports timetabling problem that abstracts the most important timetabling aspects of Major League Baseball. Given an even number of teams, pairwise distances between all venues, and two integers $L$ and $U$, the TTP consists of constructing a minimum distance $2 \mathrm{RR}$ that respects the following two hard constraints. First, the length of every road trip and home stand must be between $L$ and $U$ inclusive. Second, no two teams can play each other in two consecutive time slots.

Trick (2010) provides an online database with the best known results for several instance classes. The first class contains instances with unit distances and have up to 24 teams. For this class of instances, Urrutia and Ribeiro (2006) show that distance minimization is equivalent with break maximization. Somewhat similar, the circular distance instances (Easton et al., 2001) have up to 20 teams and embed teams' venues on a circle. Although the traveling salesman problem with a constant or circular distance matrix is trivially solvable, the traveling tournament problem remains challenging. The Galaxy instances (Uthus et al., 2012) have up to 40 teams and feature a distance matrix that is based on three-dimensional distances between several exoplanets and Earth. Four other instance classes are based on air distances between the cities of real-life tournaments: the National League of Major League Baseball (Easton et al., 2001) (up to 16 teams), the National Football League (Trick, 2010) (up to 32 teams), the Super 14 rugby cup (Uthus et al., 2009) (up to 14 teams), and the Brazilian football championship (Ribeiro and Urrutia, 2007) (24 teams). Besides, our database also provides two classes of linear distance instances from (Hoshino and Kawarabayashi, 2012), each up to 24 teams, that embed the teams' venues on a straight line. In the line-distance instances the teams venues are evenly spaced on a straight line, whereas in the increasing-distance instances the teams' venues are embedded on a line in such a way that the distance between two consecutive teams always increases with one distance unit.

Three problem variants of the TTP have been proposed for which instances can be build by using any of the above instances. First, the mirrored TTP additionally requires that the $2 \mathrm{RR}$ is mirrored (Ribeiro and Urrutia, 2007). Second, Bao and Trick (2010) propose the time-relaxed TTP in which there are arbitrarily many time slots. Byes are ignored in determining the length of a homestand or roadtrip, and in determining whether a repeater has occurred. Third, Hoshino and Kawarabayashi (2011a) propose the multi-round balanced TTP that deals with the construction of a minimum-travel kRR timetable (the classic TTP only considers $k=2$ ). For fairness reasons the timetable must additionally be phased (the authors call this balanced), and the difference in home and away games for each team must be lower than two (Nurmi et al. (2010) call this property 2-balancedness). For this problem variant, two additional instances are available with distances based on two divisions of the Nippon Professional Baseball league (NPB). In each of the two instances, there are 6 teams and $k=8$.

Furthermore, the following problem variants have been proposed that require additional data. In the TTP with predefined venues, the teams compete in a $1 \mathrm{RR}$ for which the home team of each game is given (Melo et al., 2009). In total, our database includes 20 instances with 18 teams and 20 instances with 20 teams. Somewhat similar, a non-round-robin variant exists that allows any number of games between teams (Trick, 2010). In total 
4 instances with up to 30 teams are available based on data from Major League Baseball. Finally, the bipartite traveling tournament problem (Hoshino and Kawarabayashi, 2011b) requires to construct a minimum distance 2BRR timetable. Data is available for the inter-league games of the NPB (12 teams) and the inter-league games of the National Basketball Association (30 teams).

Our repository also contains one instance involving travel minimization based on Argentina's first division volleyball league (Bonomo et al., 2012). The problem consists of minimizing the total travel distance in a compact mirrored 2RR for 12 teams, thereby considering the following six hard constraints. First, each team team is paired with another team with whom it cannot play simultaneously at home. The two teams of each pairing also have to play against each other in the first time slot of each season half. In addition, breaks are forbidden in the second time slot and the maximal length of a homestand or roadtrip is limited to two. Besides, some teams cannot play home in given time slots, there are fixed games, and the difference in the number of games played can never be larger than one. Finally, there are Thursday and Saturday time slots and it is required that each team plays half of its games on each of the two days. Data of this instance is based on (Nurmi et al., 2010), with the major modification being the reintroduction of the travel minimization as was original the case in (Bonomo et al., 2012).

\subsection{Cost and revenue optimization}

This section considers problems that assign a cost $c_{i, j, s}$ for playing game $(i, j) \in G$ on time slot $s \in S$. Given these costs, the objective is to find a feasible timetable that minimizes the sum of the scheduled games' costs. These costs can be used to model a wide variety of practical considerations (see (Briskorn and Drexl, 2009b)). As an example, costs can be used to model preferences of teams to play home or away in time slot $s$, or to model game to time slot assignment preferences. Similarly, costs can be used to maximize the overall attendance by using ticket sale estimates in combination with a maximization function.

First off, our repository includes 10 instances from (Uthus et al., 2008) with 8 to 16 teams that both require to minimize and maximize the total cost of the timetable. Costs in these instances are randomly generated, and there are no other hard constraints. Moreover, there are two instances based on the Chile's first division football tournament (Durán et al., 2007). The problem is to construct a compact 1RR with 20 teams, thereby considering 6 hard constraints. Since Chile stretches over 4,300 kilometers north to south, many of the constraints have a geographical origin. To keep game cancellations at a low level, southern teams cannot play home games in the rain season. Neither can popular teams play in outlying areas during summer since then the recording equipment must be quick to deploy for other events. Instead, popular games in the summer must be played at venues in touristic regions. Since in a $1 R R$ any two teams only meet once, it is necessary to fairly distribute the home advantage: each team plays at least nine and at most ten games at home. To further increase fairness, no team can have more than two breaks, and there cannot be a break in the last time slot. The objective is to maximize the number of attractive games near the end of the season. Our repository also includes one instance for the second division of the Chilean football league that organizes a mirrored 4RR among 11 teams (Durán et al., 2012). The instance contains ten different hard constraints: the main difference with regard to the first division is the addition of some hard constraints to balance the travel distance of teams since second division teams usually travel by bus to keep costs down. As in the first division, the objective is to maximize the number of attractive games near the end of the season.

\subsection{Carryover effects optimization}

Russell (1980) introduces the problem of constructing a feasible 1RR timetable with a minimum COE-value (no other constraints are considered). Our database includes instances and the best known solutions (obtained from Kidd (2010)) for this problem with up to 32 teams. A weighted variant of this problem is introduced in Guedes and Ribeiro (2011). They assign a weight $w_{i, j}$ to every ordered pair $(i, j)$ of teams, e.g. to represent relative strengths, and minimize the weighted COE-value. In total, our problem repository contains test instances based on four different types of weight matrices with up to 20 teams (see (Guedes and Ribeiro, 2011)). First, the random instances assign a uniformly chosen weight to each team pair. Second, the linear instances assign a strength level to each team and set the weight of a team pair equal to the absolute difference between the strength level of the two teams. Third, the perturbed linear instances additionally increase the weight generated by the linear instances with a uniformly chosen value. Last but not least, there are six instances (from 20 to 24 teams) in which the strength level of teams is determined by real-life data from the Brazilian football championship. As in the linear instances, the weight is set equal to the absolute difference between the strength level of the two teams.

Furthermore, our problem repository includes instances with 8 to 18 teams based on the Turkish major football league (see (Günneç and Demir, 2018)). The problem still requires to minimize the COE-value but this time the 
tournament in question is a mirrored 2RR. Moreover, a team cannot have more than one break per season half and there should be no breaks on the first and last time slot.

\subsection{Soft constraint optimization}

The objective in the constrained sports timetabling problem is to construct a timetable that respects all hard constraints and that minimizes the weighted sum of all soft constraint violations.

A first instance is based on the 2008-2009 season of the Finnish major ice hockey league (see (Kyngäs and Nurmi, 2009b)). The instance consists of 14 teams that play a 2RR, supplemented with two or three games per team. To facilitate the timetabling generation process there are 30 time slots, one time slot more than strictly needed, making the tournament time-relaxed. The four hard constraints involve venue availability, shared venue constraints, forbidden breaks in the second time slot, and a number of fixed games. Moreover, there are ten soft constraints. The most prominent ones enforce that two teams meet each other at home and in turn away, request at least seven time slots between two games with the same opponents, stipulate that the difference in home and away games for each team must be lower than two (i.e. the timetable is preferably 2-balanced), and request that the number of breaks per team is not larger than 6. A similar instance is based on the 2008-2009 Finnish first division ice hockey league (see Kyngäs and Nurmi (2009a)). In this instance, there are 12 teams that compete in a compact $2 \mathrm{RR}$. The three hard and six soft constraints form a subset of the ones of the major ice hockey league. Data for both instances originates from Nurmi et al. (2010) and slightly deviates from the original instances. In a follow-up study, Nurmi et al. (2015) construct the 2013-2014 season of the major ice hockey league. The main difference is that this instance consists of 14 teams that play a 4RR. Moreover, the teams are partitioned into two subgroups that each play an additional 1RR. In total the instance contains four hard constraints, and 12 soft constraints. The main addition is that the timetable should include one 'back-to-back weekend' in which teams playing against each other on Friday also play against each other on Saturday. Furthermore, due to large travel distances, some teams that play a home game on a Friday time slot cannot play away against a far away team on the next Saturday time slot. Finally, the number of games on Friday or Saturday time slots should be maximized.

Nurmi et al. (2010) also provide three instances based on the Austrian football (10 teams), the German football (18 teams), and the German handball championship (18 teams) (see Bartsch et al. (2006)). In all three instances, the problem consists of constructing a compact mirrored $2 \mathrm{RR}$ and there is only one soft constraint in which teams request not to play home in given time slots. The Austrian football instance has three hard constraints, the German handball instance has 5 hard constraints, and the German football instance has 4 hard constraints. In all three instances, the total number of breaks must be minimal. Besides, some teams share a venue in the Austrian and German football instances, and the number of simultaneous games between teams in the same strength group is limited in the German handball instance. For more details, we refer to (Nurmi et al., 2010; Bartsch et al., 2006).

The Belgian major football league organizes a compact mirrored 2RR between 18 teams (Goossens and Spieksma, 2009). A first set of two hard constraints deals with venue availability. Indeed, Belgian mayors have the right to forbid home games of teams on time slots during which they cannot guarantee public order and safety. Similarly, some teams cannot play home at the same time since they share a venue or since they are located in the same police zone. A third hard constraint states that there is at most one simultaneous game between the four strongest teams. Finally, four more hard constraints regulate the breaks: the total number of breaks must be minimal, each team has at most three breaks, no team plays more than two consecutive home or away games, and no team has a break at the start or end of the season. Besides, the league organizers impose six soft constraints. Most prominently, television broadcasters request that top games are planned during attractive time slots, and that in each time slot at least one top team plays away. In total, three instances for different seasons are available.

The instance based on the 2012 first stage of the Ecuadorian major football championship (series A) consists of a compact inverted 2RR with 12 teams (Recalde et al., 2013). Interesting is the objective to minimize the total number of breaks that are not pseudo breaks. A team has a pseudo break when it plays two consecutive away games of which at least one game is against a team from its own geographical group. In addition, the following hard constraints must be taken into account. Teams can play at most two consecutive home or away games, the total number of breaks per team is limited to two, and breaks cannot occur in the first round. Geographical constraints require that half of the teams in each region play home. Moreover, some teams share venues and so-called classic games cannot be planned during the first or last two time slots.

The Danish major football tournament organizes a compact phased 3RR with 12 teams (see Rasmussen (2008)). For this reason, this instance requires to fairly distribute the home advantage of teams' games. In total there are six hard constraints. First off, the team must play a 1RR in the first 11 time slots such that each team plays between five and six home games. Thereafter, the teams compete in a regular compact $2 \mathrm{RR}$. Another set of hard constraints regulate breaks: teams cannot play more than two consecutive home or away games, and teams cannot have a break in the last time slot. The final two hard constraints stipulate that there must be at least three 
time slots between two games with the same opponents, and that some teams cannot play home or away in certain time slots. In addition, there are 7 soft constraints that further try to balance the home advantage of the teams, minimize the total number of breaks, and balance the games per geographical region.

In total 10 instances are based on the 2013-2014 top Swedish handball tournament that organizes a two-phase tournament in which 14 teams are partitioned into two divisions that first play a compact 1RR before playing a mirrored 2RR among all teams (Larson and Johansson, 2014; Carlsson et al., 2017). Two hard constraints make that the phases must be planned in an integrated way: the total number of breaks must be minimal, and teams must meet each other at home and in turn away. Moreover, some pair of teams (within and across divisions) cannot play simultaneously at home, and the difference in home and away games for each team must be lower than two at any point in time. In total there are two soft constraints. To increase the visibility of handball the organizers request as many 'derby games' as possible in specific set of time slots. Besides, team preferences to play away should be respected at the highest extent possible.

Our repository also includes ten instances based on the Welsh major rugby tournament (Lewis and Thompson, 2011) that plays according to a compact $2 \mathrm{RR}$ with 12 to 18 teams. There are three hard constraints. To begin, some teams share a venue and must therefore not play simultaneously at home. Besides, venue availability must be respected. The tournament organizers also require two derby time slots on Christmas and Easter during which teams have to play against a local rival team. Furthermore, there are two soft constraints: breaks should be minimized, the timetable should be phased, and a pair of teams should meet at most once per five time slots.

The instance based on the 2016-2017 Italian national volleyball championship contains 14 teams that compete in a compact mirrored 2RR (Cocchi et al., 2018). In total, there are 10 hard constraints of which the most notable are the following. First, breaks must not occur during the first and last two time slots of each season half, and breaks must be separated by at least two time slots. A team can play at most two consecutive home or away games, and it should play half of its home games in the first half of the season. Similarly, some teams share a venue and the number of simultaneous games between top teams is limited. There are seven soft constraints. As an example, the number of breaks should be minimized. Since most fans can attend games on Boxing day, it is also important that teams play as close as possible to their home venue during this time slot. Somewhat similar, if a team plays consecutively away on a midweek time slot, then the two away venues should be as close as possible to the home venue of this team.

The instance based on the 2003 season of the National Basketball League in New Zealand (Wright, 2006) has three remarkable properties. First, stadium availability is highly uncertain and must be negotiated with the stadium owners. For these negotiations, a first draft timetable is produced taking into account a best guess of the availability by expressing the uncertainty with a non-availability cost. Then, the timetable is proposed to the stadium owners and the process repeats. Second, the tournament consists of a time-relaxed 2RR between 10 teams played over only 16 weeks; each week has a Friday, Saturday, and Sunday time slot. Ideally, each team plays once per week but since there are 18 games per team, each team has at least two weeks in which it plays twice (this is called a double round). In contrast to the South American world cup qualifiers (Alarcón et al., 2017), teams prefer to play twice away in such weeks since this reduces the total distance traveled. Another remarkable property is that the instance features around 15 constraints that are all soft (although some of them have a very high cost, essentially making them hard). Most important is to avoid three games per week, and to avoid that teams play home during double rounds. Moreover, it is important to minimize the venue availability cost and the total distance traveled during double rounds. We note that some idiosyncratic constraints in the instance slightly deviate from (Wright, 2006) to make the instance more general.

Schönberger et al. (2004) construct a time-relaxed 2RR for a non-professional table-tennis tournament in Germany. Teams in this tournament only have limited access to venues, and feature non-professional players. For this reason, each team provides a list of $\alpha$ time slots during which it can play a home game, and a list of $\beta$ time slots during which it cannot play any game. Besides, there are two other hard constraints. First, the tournament must be phased. Second, to increase the accuracy of the rankings, the season is subdivided into six parts of equal length during which each team must play an equal number of games. Additionally, there are two soft constraints: teams request a minimal number of byes between consecutive games, and each team should play the same number of home games in the first and second part. Schönberger et al. (2004) propose a variety of artificial instances in which there are 10 teams, and 200 time slots. In order to ensure feasibility of all instances (for both soft and hard constraints), instances are constructed around a feasible solution. Within the proposed instances, $\alpha$ varies in $\{10,15,20\}$ and $\beta$ varies in $\{0,10, \ldots, 180\}$. Our problem repository includes a selection of the 570 originally proposed instances.

Van Bulck et al. (2019) construct a time-relaxed 2RR for a non-professional indoor football tournament. Each team in this tournament also provides a list of time slots on which it can play a home game, and a list of time slots during which it cannot play any game. Besides, a team cannot play more than two games per four time slots and two teams can meet at most once per 60 time slots. In order to balance the teams' games over the season, the 
objective function penalizes the timetable each time the number of time slots between two consecutive games of a team is less then three time slots. In total, Van Bulck et al. (2019) propose 53 instances based on real-life data from a local non-professional indoor football league. In all instances, the size of the team set varies between 13 and 15 and there are either 273 or 274 time slots (a time-constrained timetable would require between 26 and 30 time slots). On average, teams indicate that they can play a home game on 4.5 time slots more than the number of opponents in the tournament, and that they cannot play any game on 14.8 time slots. In contrast to the problem description in our problem repository, the original formulation allowed not to schedule a game but this resulted in a high penalty value. From the original 53 instances, our repository does not include 9 instances since they are unfeasible if scheduling all games is a hard constraint.

\subsection{Multi-league timetabling}

Many sports tournaments partition teams into multiple leagues (also called divisions) such that teams only play games with teams from their own league. The partitioning of the teams might be based on geographical location or skill set, and promotion and relegation is usually strictly defined. The determination of a timetable for the different leagues is not trivial due to the large number of inter-league constraints. As an example, police might enforce that two teams located in the same city cannot play simultaneously at home. Many professional tournaments avoid inter-league conflicts by employing a sequential timetabling generation scheme: first the timetable of the highest ranked league is scheduled after which the resulting dependencies are propagated to all lower-ranked leagues. However, in most non-professional leagues, this sequential scheme is not very practical since it does not fully exploit scarce resources and no single league should be given priority over the other leagues.

Schönberger (2017) proposes the following multi-league timetabling variant of the non-professional table tennis tournament described in (Schönberger et al., 2004). First off, the tournament consists of 23 clubs that delegate teams to three different leagues. More precisely, two of the clubs have exactly one team in each league, three clubs have one team in two uniformly chosen leagues, and the other 18 clubs delegate one team to each league such that each league features exactly 10 teams. Each club $c$ has $n_{c}$ venues on which the club's teams can play home games. Each team also provides a list of $\alpha$ time slots during which it can play home, and a list of $\beta$ time slots during which it cannot play any game. In total, there are 90 time slots on which games can be played. The problem requires to construct for each of the three leagues a phased $2 \mathrm{RR}$ timetable that respects the following three hard constraints. First off, a team can only play home if its venue is available. Moreover, a team can only play a game if it is available. Besides, at $\operatorname{most} n_{c}$ teams of club $c$ can simultaneously play home in order to respect venue capacity. Similarly, the $i$-th team of a club cannot play simultaneously with the $i+1$-th team of a club since these two teams must be able to substitute players. The only soft constraint requests that a team has at least one bye between two consecutive games. Our problem repository contains numerous instances of this problem for varying levels of $\alpha \in\{5,7, \ldots, 15\}$ and $\beta \in\{0,2, \ldots, 10\}$. For an in-depth discussion of how these instances are generated, we refer to Schönberger (2017).

Kendall (2008) proposes four instances that consider the problem of timetabling the games in all four different leagues of the English football tournament that are played on Boxing day and New Year's day. In all the considered instances, 20 teams participate to the highest league, whereas the other three leagues feature 24 teams each. During the two time slots, each team must play one home game and one away game such that the two opponents of each team are different, and that some pair of teams do not meet at all. This is not an easy task since 'clash' constraints limit the number of simultaneous home games of teams that are geographically close. In all three leagues, the objective is to minimize the total distance traveled by the teams, thereby assuming that teams return home after playing an away game.

\section{Conclusion}

In this paper, we collected artificial and real-life sports timetabling instances from over 15 different countries and eight different sports. We described the main instance properties and converted all problem instances into the XML format of RobinX. First of all, this transformation shows the flexibility of RobinX being able to classify all considered instances. Second, the resulting database, which is unprecedented in size, offers researchers an opportunity to benchmark their algorithms on problem instances other than the specific sports league under consideration. This should boost algorithmic performance since more general insights can be deduced. With this paper, we invite researchers to submit new problem instances and solutions. 


\section{References}

Alarcón, F., Durán, G., Guajardo, M., Miranda, J., Muñoz, H., Ramírez, L., Ramírez, M., Sauré, D., Siebert, M., Souyris, S., et al. (2017). Operations research transforms the scheduling of Chilean soccer leagues and South American world cup qualifiers. Interfaces, 47:52-69.

Ball, B. C. and Webster, D. B. (1977). Optimal scheduling for even-numbered team athletic conferences. Aiie T., 9:161-169.

Bao, R. and Trick, M. (2010). The relaxed traveling tournament problem. In McCollum, B., Burke, E., and White, G., editors, Proceedings of the 8th International Conference on the Practice and Theory of Automated Timetabling, pages 472-476, Belfast. PATAT.

Bartsch, T., Drexl, A., and Kröger, S. (2006). Scheduling the professional soccer leagues of Austria and Germany. Comput. Oper. Res., 33:1907-1937.

Bonomo, F., Cardemil, A., Durán, G., Marenco, J., and Sabán, D. (2012). An application of the traveling tournament problem: The Argentine volleyball league. Interfaces, 42(3):245-259.

Briskorn, D. (2009). Combinatorial properties of strength groups in round robin tournaments. Eur. J. Oper. Res., 192:744 - 754 .

Briskorn, D. and Drexl, A. (2009a). A branching scheme for finding cost-minimal round robin tournaments. Eur. J. Oper. Res., 197:68-76.

Briskorn, D. and Drexl, A. (2009b). IP models for round robin tournaments. Comput. Oper. Res., 36:837 - 852.

Carlsson, M., Johansson, M., and Larson, J. (2017). Scheduling double round-robin tournaments with divisional play using constraint programming. Eur. J. Oper. Res., 259:1180-1190.

Cocchi, G., Galligari, A., Nicolino, F. P., Piccialli, V., Schoen, F., and Sciandrone, M. (2018). Scheduling the Italian national volleyball tournament. Interfaces, 48:271-284.

Della Croce, D. and Oliveri, D. (2006). Scheduling the Italian Football League: an ILP-based approach. Comput. Oper. Res., 33:1963 - 1974. Special Issue: Operations Research in Sport.

Durán, G., Guajardo, M., Miranda, J., Sauré, D., Souyris, S., Weintraub, A., and Wolf, R. (2007). Scheduling the Chilean soccer league by integer programming. Interfaces, 37:539-552.

Durán, G., Guajardo, M., and Sauré, D. (2017). Scheduling the South American qualifiers to the 2018 FIFA World Cup by integer programming. Eur. J. Oper. Res., 262:1109- 1115.

Durán, G., Guajardo, M., and Wolf-Yadlin, R. (2012). Operations research techniques for scheduling Chile's second division soccer league. Interfaces, 42:273-285.

Easton, K., Nemhauser, G., and Trick, M. (2001). The traveling tournament problem description and benchmarks. In Walsh, T., editor, Principles and Practice of Constraint Programming - CP 2001, pages 580-584, Berlin, Heidelberg. Springer.

Goossens, D. and Spieksma, F. (2009). Scheduling the Belgian soccer league. Interfaces, 39:109-118.

Goossens, D. and Spieksma, F. C. (2012). The carryover effect does not influence football results. J. Sport. Econ., 13:288-305.

Guedes, A. C. B. and Ribeiro, C. C. (2011). A heuristic for minimizing weighted carry-over effects in round robin tournaments. J. Sched., 14:655-667.

Günneç, D. and Demir, E. (2018). Fair-fixture: Minimizing carry-over effects in football leagues. Journal of Industrial \& Management Optimization, 13:1-16.

Hausken, M. D., Andersson, H., Fagerholt, K., and Flatberg, T. (2012). Retracted: Scheduling the Norwegian football league. Int. T. Oper. Res., 20:59-77.

Hoshino, R. and Kawarabayashi, K. (2011a). A multi-round generalization of the traveling tournament problem and its application to Japanese baseball. Eur. J. Oper. Res., 215:481 - 497. 
Hoshino, R. and Kawarabayashi, K. (2011b). Scheduling bipartite tournaments to minimize total travel distance. J. Artif. Intell. Res., 42:91-124.

Hoshino, R. and Kawarabayashi, K. (2012). Generating approximate solutions to the traveling tournament problem using a linear distance relaxation. J. Artif. Intell. Res., 45:257-286.

Kendall, G. (2008). Scheduling English football fixtures over holiday periods. J. Oper. Res. Soc., 59:743-755.

Kendall, G., Knust, S., Ribeiro, C. C., and Urrutia, S. (2010). Scheduling in sports: An annotated bibliography. Comput. Oper. Res., 37:1-19.

Kidd, M. P. (2010). A tabu-search for minimising the carry-over effects value of a round-robin tournament. ORiON, 26:125-141.

Kingston, J. H., Post, G., and Vanden Berghe, G. (2018). A unified nurse rostering model based on XHSTT. In Burke, E. K., Di Gaspero, L., McCollum, B., Musliu, N., and Özcan, E., editors, Proceedings of the 12th International Conference on the Practice and Theory of Automated Timetabling, pages 81-96, Vienna. PATAT.

Knust, S. (2018). Classification of literature on sports scheduling. http://www2.inf.uos.de/knust/ sportssched/sportlit_class/. Accessed: 04/12/2018.

Kyngäs, J. and Nurmi, K. (2009a). Scheduling the Finnish 1st division ice hockey league. In Proceedings of the twenty-second Florida Artificial Intelligence Research Society Conference, pages 195-200, Florida. AAAI Press.

Kyngäs, J. and Nurmi, K. (2009b). Scheduling the Finnish Major Ice Hockey League. In Computational Intelligence in Scheduling, 2009. CI-Sched'09. IEEE Symposium on, pages 84-89. IEEE.

Larson, J. and Johansson, M. (2014). Constructing schedules for sports leagues with divisional and round-robin tournaments. Journal of Quantitative Analysis in Sports, 10:119-129.

Lewis, R. and Thompson, J. (2011). On the application of graph colouring techniques in round-robin sports scheduling. Comput. Oper. Res., 38:190 - 204. Project Management and Scheduling.

Melo, R. A., Urrutia, S., and Ribeiro, C. C. (2009). The traveling tournament problem with predefined venues. $J$. Sched., 12:607.

Nemhauser, G. L. and Trick, M. A. (1998). Scheduling a major college basketball conference. Oper. Res., 46:1-8.

Nurmi, K., Goossens, D., Bartsch, T., Bonomo, F., Briskorn, D., Durań, G., Kyngäs, J., Marenco, J., Ribeiro, C. C., Spieksma, F., Urrutia, S., and Wolf, R. (2010). A framework for scheduling professional sports leagues. In Katagir, H., Xu, L., and Chan, A. H., editors, Ao,S-I., volume 5, page 1428. Springer.

Nurmi, K., Goossens, D., and Kyngäs, J. (2013). Scheduling a triple round robin tournament with minitournaments for the Finnish national youth ice hockey league. J. Oper. Res. Soc., 65:1770-1779.

Nurmi, K., Kyngäs, J., Goossens, D., and Kyngäs, N. (2015). Scheduling the Finnish Major Ice Hockey League using the PEAST algorithm. In Transactions on Engineering Technologies, pages 155-168. Springer.

Post, G., Ahmadi, S., Daskalaki, S., Kingston, J. H., Kyngäs, J., Nurmi, C., and Ranson, D. (2012). An XML format for benchmarks in high school timetabling. Ann. Oper. Res., 194:385-397.

Post, G., Kingston, J. H., Ahmadi, S., Daskalaki, S., Gogos, C., Kyngäs, J., Nurmi, C., Musliu, N., Pillay, N., Santos, H., et al. (2014). XHSTT: an XML archive for high school timetabling problems in different countries. Ann. Oper. Res., 218:295-301.

Rasmussen, R. V. (2008). Scheduling a triple round robin tournament for the best Danish soccer league. Eur. J. Oper. Res., 185:795 - 810.

Recalde, D., Torres, R., and Vaca, P. (2013). Scheduling the professional Ecuadorian football league by integer programming. Comput. \& Oper. Res., 40:2478- 2484.

Ribeiro, C. C. and Urrutia, S. (2007). Heuristics for the mirrored traveling tournament problem. Eur. J. Oper. Res., 179:775-787. 
Russell, K. G. (1980). Balancing carry-over effects in round robin tournaments. Biometrika, 67:127-131.

Schönberger, J. (2017). The championship timetabling problem-construction and justification of test cases. In Proceedings of MathSport International 2017 Conference, page 330. MathSport.

Schönberger, J., Mattfeld, D. C., and Kopfer, H. (2004). Memetic algorithm timetabling for non-commercial sport leagues. Eur. J. Oper. Res., 153:102-116.

Trick, M. (2010). Challenge traveling tournament instances. http://mat.gsia.cmu. edu/TOURN/. Accessed: $05 / 08 / 2018$.

Urrutia, S. and Ribeiro, C. C. (2006). Maximizing breaks and bounding solutions to the mirrored traveling tournament problem. Discrete Appl. Math., 154:1932-1938.

Uthus, D. C., Riddle, P. J., and Guesgen, H. W. (2008). Ant colony optimization and the single round robin maximum value problem. In Dorigo, M., Birattari, M., Blum, C., Clerc, M., Stützle, T., and Winfield, A. F. T., editors, Ant Colony Optimization and Swarm Intelligence, pages 243-250, Berlin. Springer.

Uthus, D. C., Riddle, P. J., and Guesgen, H. W. (2009). DFS* and the traveling tournament problem. In van Hoeve, W.-J. and Hooker, J. N., editors, Integration of AI and OR Techniques in Constraint Programming for Combinatorial Optimization Problems, pages 279-293, Berlin, Heidelberg. Springer.

Uthus, D. C., Riddle, P. J., and Guesgen, H. W. (2012). Solving the traveling tournament problem with iterativedeepening A*. J. Sched., 15:601-614.

Van Bulck, D., Goossens, D., Schönberger, J., and Guajardo, M. (2020). Robinx: A three-field classification and unified data format for round-robin sports timetabling. Eur. J. Oper. Res., 280:568 - 580.

Van Bulck, D., Goossens, D. R., and Spieksma, F. C. R. (2019). Scheduling a non-professional indoor football league: a tabu search based approach. Ann. Oper. Res., 275:715-730.

Wright, M. (2006). Scheduling fixtures for basketball New Zealand. Comput. \& Oper. Res., 33:1875 - 1893. Special Issue: Operations Research in Sport.

Zhang, H. (2002). Generating college conference basketball schedules by a SAT solver. In Proceedings of the fifth international symposium on the theory and applications of satisfiability testing, pages 281-291. 